CORRIGENDUM

doi:10.1038/nature10459

\title{
Detection of prokaryotic mRNA signifies microbial viability and promotes immunity
}

Leif E. Sander, Michael J. Davis, Mark V. Boekschoten, Derk Amsen, Christopher C. Dascher, Bernard Ryffel,

Joel A. Swanson, Michael Müller \& J. Magarian Blander

Nature 474, 385-389 (2011).

In Fig. 1d of this Letter, the labels HKEC and EC were swapped in the print version. The lane labelled HKEC should be labelled EC and the lane labelled EC should be labelled HKEC. The error has been corrected online in the HTML and PDF versions. 